\title{
Functional Connectivity in Brain Networks Underlying Cognitive Control in Chronic Cannabis Users
}

\author{
Ian H Harding*,', Nadia Solowij ${ }^{2,3}$, Ben J Harrison', Michael Takagi ', Valentina Lorenzetti', Dan I Lubman ${ }^{4}$, \\ Marc L Seal ${ }^{5,6}$, Christos Pantelis' and Murat Yücel*,I \\ 'Department of Psychiatry, Melbourne Neuropsychiatry Centre, University of Melbourne and Melbourne Health, Melbourne, VIC, Australia; \\ ${ }^{2}$ School of Psychology, University of Wollongong, Wollongong, NSW, Australia; ${ }^{3}$ Schizophrenia Research Institute, Sydney, NSW, Australia; \\ ${ }^{4}$ Turning Point Alcohol and Drug Centre, Eastern Health and Monash University, Melbourne, VIC, Australia; ${ }^{5}$ Murdoch Childrens Research \\ Institute, Melbourne, VIC, Australia; ${ }^{6}$ Department of Paediatrics, University of Melbourne, Melbourne, VIC, Australia
}

\begin{abstract}
The long-term effect of regular cannabis use on brain function underlying cognitive control remains equivocal. Cognitive control abilities are thought to have a major role in everyday functioning, and their dysfunction has been implicated in the maintenance of maladaptive drug-taking patterns. In this study, the Multi-Source Interference Task was employed alongside functional magnetic resonance imaging and psychophysiological interaction methods to investigate functional interactions between brain regions underlying cognitive control. Current cannabis users with a history of greater than 10 years of daily or near-daily cannabis smoking $(n=21)$ were compared with age, gender, and IQ-matched non-using controls $(n=21)$. No differences in behavioral performance or magnitude of task-related brain activations were evident between the groups. However, greater connectivity between the prefrontal cortex and the occipitoparietal cortex was evident in cannabis users, as compared with controls, as cognitive control demands increased. The magnitude of this connectivity was positively associated with age of onset and lifetime exposure to cannabis. These findings suggest that brain regions responsible for coordinating behavioral control have an increased influence on the direction and switching of attention in cannabis users, and that these changes may have a compensatory role in mitigating cannabis-related impairments in cognitive control or perceptual processes.
\end{abstract}

Neuropsychopharmacology (2012) 37, 1923-1933; doi:I0.1038/npp.2012.39; published online 25 April 2012

Keywords: attention; brain; cannabis; cognitive control; functional connectivity

\section{INTRODUCTION}

Little is yet known of the long-term consequences of cannabis use on brain function underlying cognitive control abilities. Cognitive control processes promote adaptive and dynamic goal-directed behavior by biasing perceptual and information processing within the brain in favor of stimuli and responses relevant to current goals (Botvinick et al, 2001; Ridderinkhof et al, 2004). These processes include the ability to focus and shift attention, to inhibit inappropriate behavioral responses, and to monitor and change one's behavior in response to ongoing environmental feedback (MacDonald et al, 2000; Ridderinkhof et al, 2004; Suchy, 2009). Cognitive control processes support healthy social

\footnotetext{
*Correspondence: Dr IH Harding and Professor M Yücel, Department of Psychiatry, Melbourne Neuropsychiatry Centre, Alan Gilbert Building, University of Melbourne, 3/16I Barry Street, Carlton, Melbourne, VIC 3053, Australia, Tel: (+61 3) 8344 186I, Fax: (+61 3) 93480469 , E-mail: hardingi@unimelb.edu.au and murat@unimelb.edu.au Received 21 September 2011; revised 13 February 2012; accepted I March 2012
}

functioning, educational achievement, vocational performance, and activities of daily living (Cahn-Weiner et al, 2007; Henry et al, 2009; Suchy, 2009; Williams et al, 2009). Their impairment, on the other hand, has been linked to the initiation and maintenance of addictive behavior by compromising the effective inhibition of drug-seeking behavior (Fillmore, 2003; Koob and Volkow, 2010; Lubman et al, 2004). Elucidating the impact of cannabis use on cognitive control and underlying brain function is therefore relevant to developing appropriate interventions for those with cannabis-use disorders and for further understanding the biological mechanisms governing substance use and abuse.

Available evidence regarding the existence and behavioral significance of cognitive control impairments, and associated abnormalities in underlying neural correlates, remains equivocal in cannabis-using populations. Neuropsychological studies employing tasks of cognitive-interference resolution, selective attention, and inhibitory control have reported relatively normal or minimally impaired performance in chronic cannabis users assessed in the non-intoxicated state (Fernandez-Serrano et al, 2010; Lyons et al, 2004; Pope et al, 
2001; Solowij et al, 2002; Takagi et al, 2010; Verdejo-Garcia et al, 2005). Recent neuroimaging investigations, however, have described functional disruptions within the brain during the performance of cognitive control tasks, both concurrent with (Abdullaev et al, 2010; Battisti et al, 2010), and in the absence of behavioral deficits (Eldreth et al, 2004; Hester et al, 2009; Tapert et al, 2007) in similar samples of chronic users. These aberrations have largely been reported within brain regions comprising the cognitive control network (Cabeza and Nyberg, 2000; Nee et al, 2007), including the anterior cingulate, lateral prefrontal, and lateral posterior parietal cortices. However, considerable variability in the laterality, anatomical loci, and even valence (ie, hyper- $v s$ hypo-activation) of reported functional abnormalities exists between studies. Impairments may therefore best be described as subtle, targeted to select cognitive domains, and/or dependent on the exact cohort under study.

In the investigation of cannabis-related effects on brain function underlying cognitive control, the current study conferred several advantages to previous research. First, the investigation of non-treatment seeking adults with a longterm and heavy exposure to cannabis, alongside minimal exposure to other drugs and no comorbid mental health issues, increased sensitivity to the biological correlates of cannabis use. Second, by employing the Multi-Source Interference Task (Bush and Shin, 2006), multiple domains of cognitive control, including interference resolution and selective attention, were simultaneously taxed, increasing the opportunity to identify subtle and/or specific disruptions. Finally, in addition to investigating the magnitude of taskinduced brain activations, the integrity of functional interrelationships between brain regions mediating cognitive control was also examined. Increased cognitive control demands have been linked with dynamic increases in functional connectivity between regions forming the cognitive control network (Egner and Hirsch, 2005a, b; Fan et al, 2008; Wang et al, 2010), whereas both abnormal increases and decreases in this connectivity have been linked to behavioral impairments in a variety of clinical disorders (eg, Konrad and Eickhoff, 2010; Pettersson-Yeo et al, 2011; Rowe, 2010). Investigating the impact of cannabis on both activation and connectivity is particularly compelling, given the high density of endocannabinoid receptors located throughout the cognitive control network (Eggan and Lewis, 2007) and the well-documented role these receptors have in the modulation of synaptic efficacy (Chevaleyre et al, 2006; Kano et al, 2009). In one such study, Jacobsen et al (2007) identified reduced connectivity between the left inferior parietal cortex and the anterior cingulate, lateral prefrontal, and insular cortices, during performance of a verbal working memory task in abstinent adolescent cannabis users. However, to our knowledge, studies of functional connectivity underlying cognitive control have yet to be undertaken.

On the basis of the existing literature examining cognitive control deficits in cannabis users discussed above, we did not predict impaired behavioral performance on the MSIT paradigm in this population. However, abnormal functional activations throughout the cognitive control network, including the anterior cingulate, lateral prefrontal, anterior insular, and posterior parietal cortices were predicted. Moreover, abnormal connectivity within this network, as well as between these regions and additional task-relevant brain areas, including the extrastriate cortices, cerebellum, and basal ganglia, were also hypothesized. Two-sided hypotheses were adopted, as the expectation of directionality cannot be adequately justified at present, given the variability between existing reports of functional impairments in similar tasks and cohorts, a current lack of comparable connectivity-based research, and evidence supporting both increased and decreased activation/connectivity underlying cognitive control deficits in psychiatric and neurological disorders. However, an earlier age of onset and heavier lifetime cannabis use were hypothesized to positively correlate with greater abnormalities in functional activations and connectivity.

\section{MATERIALS AND METHODS}

\section{Participants}

Current cannabis users who had smoked on a daily or neardaily basis for no less than 10 years $(n=21)$, and age, gender, and full-scale IQ-matched non-users who were naive or had minimal lifetime exposure to cannabis $(n=21$; Table 1) were recruited from the general community. Substance-use history was assessed by a structured interview that incorporated the Alcohol Use Disorders Identification Test (Allen et al, 1997) and a Time-Line Follow-Back procedure (Maisto et al, 1982). All cannabis users were abstinent for no less than $12 \mathrm{~h}$ before testing, based on selfreport and corroborated by analysis of urine and saliva samples (Table 1), minimizing the prevalence of acute subjective and cognitive effects of intoxication (Curran et al, 2002; Fant et al, 1998; Ramaekers et al, 2009). Urinalysis also served to rule out other concurrent recreational drug use. Additionally, all cannabis users endorsed only mild, if any, symptoms of withdrawal as measured using the Marijuana Withdrawal Checklist (Budney et al, 1999). The groups were matched for current average monthly alcohol use, but cannabis-using participants smoked a greater number of daily tobacco cigarettes. The median number of cumulative lifetime episodes of other illicit drug use ranged from 0 to 6.5 for each of amphetamines, benzodiazepines, cocaine, ecstasy, hallucinogens, inhalants, and opiates, and no participant reported regular consumption ( $>1$ use/month for any period of time) of any substance other than cannabis within the past 2 years. The age of onset of regular cannabis use, total estimated joints consumed over the lifetime, and concentrations of urinary and salivary cannabinoids at the time of testing were the primary substance-use measures examined in relation to performance and imaging measures.

All participants were right-handed, had normal or corrected-to-normal visual acuity, and were screened for past and present psychiatric or neurological illness, substance dependence (excepting cannabis dependence in the cannabis group), current use of psychotropic medication, or history of significant head injury, by structured interview including the Structured Clinical Interview for DSM-IV Axis I Disorders. This study was approved by the local Human Research and Ethics Board, and all participants provided written, informed consent before participation. 
Table I Demographic and Substance Use Measures

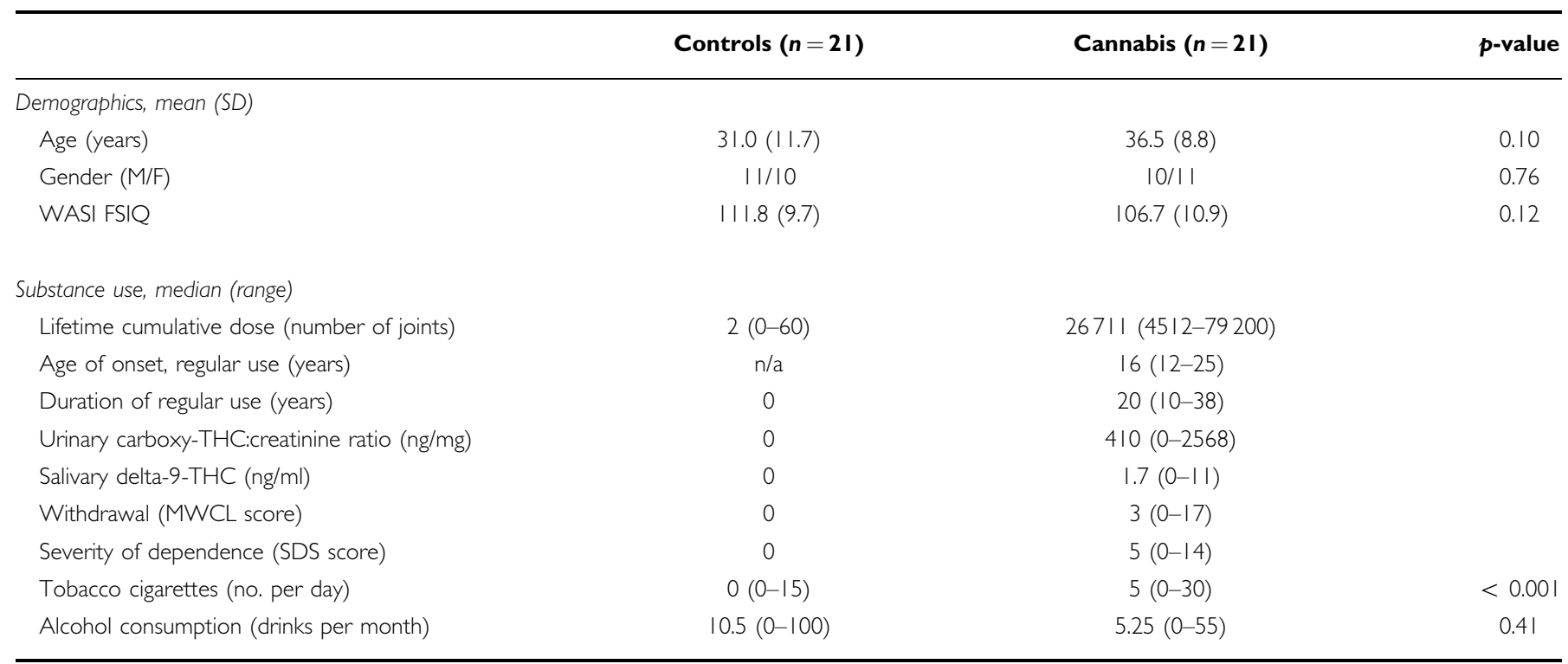

Abbreviations: MWCL, Marijuana Withdrawal Checklist; THC, tetrahydrocannabinol; WASI, Wechsler-Abbreviated Scale of Intelligence.

\section{Experimental Design}

The Multi-Source Interference Task (MSIT), a well-validated paradigm that provides for robust assay of cognitive control performance and associated neural correlates (Bush and Shin, 2006), was utilized. In brief, participants were visually presented with stimuli consisting of three numbers, or triplets, aligned horizontally. The value of each number ranged from 0 to 3 , with one number always unique (the 'target') to the other two (the 'distracters'; Figures 1a and b). Participants were instructed to identify the value of the target number by pressing one of three available buttons on an MR-compatible response box, using the first three fingers of the right (dominant) hand. Two active conditions were created by manipulating the composition of the triplets: during congruent trials (Figure 1a), the target number was always spatially aligned with the position of the correct button response (ie, a target of ' 1 ' would be presented as the left-most number) and was accompanied by two zeros (which do not represent a response alternative); during incongruent trials (Figure 1b), the target digit was always spatially misaligned and accompanied by distracters that represented an alternative response possibility. Each triplet was presented for $2000 \mathrm{~ms}$ with an inter-stimulus interval of $500 \mathrm{~ms}$. Both reaction time (RT) and accuracy were emphasized and recorded.

Correct and efficient responses to incongruent trials relative to congruent trials thus relied on: (i) selective attention mechanisms to filter irrelevant information; (ii) inhibition of prepotent responses to salient, yet incorrect response alternatives; (iii) resolution of response-based conflict created by spatial stimulus-response incongruence (ie, 'Simon' interference; Simon and Berbaum, 1990); and (iv) resolution of stimulus-based conflict created by distracter stimuli (ie, 'Flanker' interference; Eriksen and Eriksen, 1974).

The experiment followed an A-R-B-R block design (Figure 1c), where $\mathrm{A}$ and $\mathrm{B}$ represent active task conditions presented in 30-s blocks, separated by $15-\mathrm{s}$ resting periods

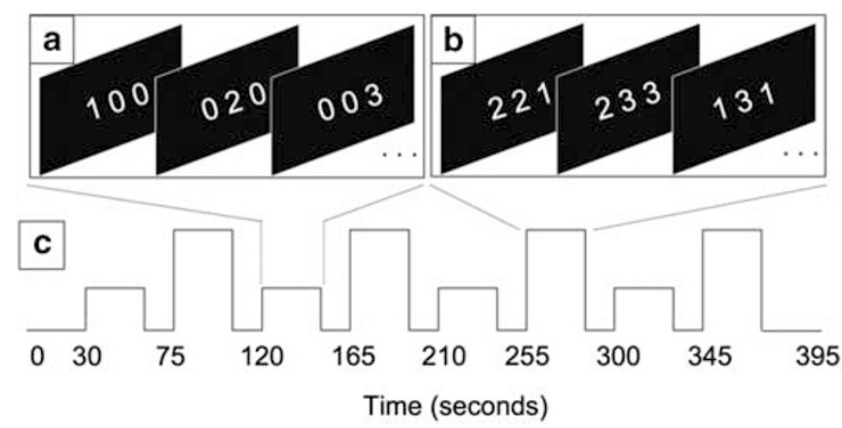

Figure I The Multi-Source Interference Task. Examples of congruent (a) and incongruent (b) stimuli, with the correct response 'I', '2', and ' 3 ' corresponding to the left, middle, and right panels, respectively. The experimental time course (c) depicts the alternating block design with interleaved passive rest periods between each task-active block.

(R), during which a fixation cross was displayed. Four blocks of each condition, containing 12 stimuli each, were presented across a single 6.5-min run. A short practice run consisting of one congruent and one incongruent block preceded scanning. Stimuli were generated and responses recorded using Presentation software (version 14.4, http:// www.neurobs.com/). Stimuli were back-projected onto a semi-transparent display at the foot of the scanner bed and viewed through a head-coil mounted mirror.

\section{Image Acquisition}

All images were obtained on a 3T Siemens Trio scanner equipped with a 32-channel head coil at the Murdoch Childrens Research Institute, Melbourne, VIC, Australia. Functional data consisted of 157 whole-brain gradient-echo echo-planar images consisting of 36 interleaved, contiguous axial slices $\left(\mathrm{TR}=2400 \mathrm{~ms} ; \mathrm{TE}=40 \mathrm{~ms}\right.$; flip angle $=90^{\circ}$; thickness $=3.0 \mathrm{~mm}$; in-plane resolution $=3.3 \mathrm{~mm}^{2}$; FOV $=$ $210 \mathrm{~mm}^{2}$ ). Three additional whole-brain volumes were 
acquired before this functional acquisition to allow magnetization to reach a steady state. High-resolution T1-weighted MPRAGE structural images were acquired for subsequent co-registration (176 sagittal slices; $1.0 \mathrm{~mm}^{3}$ isotropic voxels; $\mathrm{TR}=1900 \mathrm{~ms} ; \mathrm{TE}=2.15 \mathrm{~ms} ; \mathrm{FOV}=256 \mathrm{~mm}$ ).

\section{Behavioral Data Analysis}

Mean RT data from all correct responses were entered into a $2 \times 2$ mixed-design ANCOVA, treating task condition (congruent $v s$ incongruent) as the within-subject factor, and group (cannabis user $v s$ control) as the between-subject factor. Although the groups were matched for age and alcohol consumption, both were added to the model as covariates to control additional residual variance.

To measure the impact of tobacco use, which differed significantly between groups (regular smokers: 1 of 21 controls $v s 16$ of 21 cannabis users), the cannabis group was divided categorically into low $(n=11$, mean $(\mathrm{SD})=1.6(2.0)$ cigarettes per day) and high ( $n=10,13.7$ (6.6) cigarettes per day) tobacco users. There were no demographic or drug-use differences between these subgroups. The potential effects of past polydrug abuse were similarly assessed by dividing the cannabis group into those with $(n=10)$ and without $(n=11)$ past abuse of alternate illicit substance(s), defined as $>1$ use/month for any period of time. These subgroups did not differ on demographic or cannabis use metrics, with the exception of a greater concentration of urinary cannabinoid metabolites observed in those with a history of polydrug abuse $\left(t_{6.7}=2.43, p=0.047\right)$. However, this latter effect did not survive correction for multiple comparisons and appeared to be driven by one outlier subject. Differences in the condition effect between these categorically defined subgroups and the control cohort were assessed using one-way ANOVAs.

Multiple regression models were additionally employed in the cannabis group to assess relationships between behavioral performance and (i) lifetime cannabis use indices, including cumulative cannabis exposure and age of onset of regular cannabis use; (ii) recent cannabis use indices, including salivary and urine cannabinoid concentrations (assessed in separate models due to correlation); and (iii) acute withdrawal symptoms, as assessed by the Marijuana Withdrawal Checklist. All analyses were undertaken using SPSS (version 18.0).

Although the group-by-condition interaction effect was of primary interest, the main condition effect, collapsed across groups, was also assessed to ensure a significant behavioral effect was elicited by the task. Mean error rates across both conditions (and their log-transformed equivalents) failed to meet assumptions of normality, as expected due to ceiling effect, and were therefore analyzed using equivalent nonparametric methods (Wilcoxon signed rank, Mann-Whitney $U$, and Kruskal-Wallis tests).

\section{Functional Magnetic Resonance Imaging (fMRI) Regional Analysis}

All functional analysis was performed using SPM8 software (http://www.fil.ion.ucl.ac.uk/spm/). For each subject, the functional images were first spatially aligned to the first volume of the run using a six-parameter rigid-body transformation to correct for head movement. The anatomical scans across all participants were then used to create a study-unique group template using a diffeomorphic registration algorithm designed to improve between-subject registration (DARTEL; Ashburner, 2007). This intermediate group template was normalized to MNI space, and subsequent transformation parameters from both steps, estimated individually for each subject, applied to the motion-corrected, coregistered functional data. Voxel sizes were resampled to $2 \mathrm{~mm}^{3}$ during normalization, and images spatially smoothed using a Gaussian kernel of $8 \mathrm{~mm}$ at FWHM.

The congruent and incongruent task periods were coded as individual regressors within a general linear model (GLM) by first specifying the onset and duration of each task epoch, followed by convolution of the model with a canonical hemodynamic response function to form a model of the predicted task-related BOLD response. A $128 \mathrm{~s}$ high-pass filter was applied to remove low-frequency noise, and temporal autocorrelations were estimated using a firstorder autoregressive model. Resulting regression parameter estimates from each individual were used as summary statistics for group-level random-effects analysis entirely analogous to the 'Behavioral Data Analysis' described above.

Functional masks for between-group analyses were formed using the global conjunction of the whole-brain-positive effect of condition (incongruent $>$ congruent) from each group, thresholded at $p<0.05$ uncorrected. The use of global conjunctions and liberal thresholds ensured inclusion of all task-relevant regions across both groups. Voxelwise statistical parametric maps (SPMs; ie, T-statistic maps) inclusively restricted to these regions were then calculated for group-by-condition interactions and cannabis-use regressions using a voxelwise significance threshold of $p<0.001$ uncorrected, with a 10-voxel minimum cluster extent $\left(\mathrm{K}_{\mathrm{E}}\right)$.

\section{fMRI Connectivity Analysis}

Group differences in task-relevant functional connectivity were analyzed by assessing psychophysiological interactions (PPIs). A PPI indexes task-induced changes in the strength of connectivity between two brain regions, as measured by a change in the magnitude of the linear regression slope between their underlying activities. Significant PPIs indicate dynamic neuromodulation in response to contextual demands, and identify functional interactions that are relevant to the current task demands (Friston et al, 1997). Connectivity between each of the seven regions of interest, located in the lateral prefrontal (PFC), anterior insula (aIns), and posterior parietal cortices (PPC) bilaterally, as well as the midline dorsal anterior cingulate cortex (dACC), and all other task-relevant brain regions were assessed for between-group differences. Spheres having a $3-\mathrm{mm}$ radius were defined around the maximum positive main effect of condition for each subject within each region of interest, from which the average time series (first eigenvariate) of activity was extracted. At the individual level, three regressors were created in a GLM representing the deconvolved time course of activity in the seed region (the physiological factor), the task model (the psychological factor), and their cross-product (the PPI), respectively. The voxelwise regression estimates from the interaction term acted as summary statistics that were subsequently entered into one-way ANCOVAs, controlling 
for potential confounds as described above. Voxelwise SPMs restricted to task-relevant regions (using inclusive masks, as described above; threshold $\left.=p<0.001, K_{\mathrm{E}} \geqslant 10\right)$ were used to explore group differences in connectivity and associations between cannabis-use measures and connectivity.

\section{RESULTS}

\section{Behavior}

Analysis of RTs revealed a significant main effect of condition $\left(F_{1,38}=21.88, p<0.001\right)$, but a nonsignificant groupby-condition interaction $\left(F_{1,38}=2.47, p=0.12\right)$, while covarying for alcohol consumption and current age. No group differences due to history of other illicit drug abuse $\left(F_{2,37}=1.22, p=0.31\right)$ or tobacco smoking $\left(F_{2,37}=1.23, p=\right.$ 0.31 ) were in evidence.

Similar results were found for error rate (main effect of condition: Wilcoxon signed rank test, $Z=-4.34, p<0.001$; group-by-condition interaction: Mann-Whitney $U$-test, $Z=-1.72, p=0.085)$. Although covariates could not be directly included in the models, Spearman correlations between each of alcohol consumption and current age indicated nonsignificant relationships with the condition effect $(p>0.27)$. History of alternate illicit drug abuse (Kruskal-Wallis, $\left.\chi^{2}=3.69, p=0.16\right)$ and tobacco use $\left(\chi^{2}=3.27, p=0.20\right)$ was also not predictive of error rate.

Taken together, the task was observed to elicited robust behavioral effects with no significant performance differences apparent between groups. See Table 2 for behavioral results.

Evaluation of relationships between lifetime cannabis-use measures and RTs (incongruent-congruent) also failed to support correlations with either of lifetime cannabis dose $\left(t_{17}=-0.53, p=0.61\right)$ or age of regular cannabis-use onset $\left(t_{17}=0.72, p=0.61\right)$. Additionally, neither salivary $\left(t_{18}=0.21\right.$, $p=0.83)$ nor urinary $\left(t_{15}=0.50, p=0.63\right)$ cannabinoid measures, indexing recent cannabis use, or self-reported symptoms of withdrawal $\left(t_{19}=-1.59, p=0.13\right)$ were predictive of task effect in the cannabis group.

\section{fMRI: Regional}

Significant main effects of condition (collapsed across groups) in a priori regions of interest were observed at statistical thresholds corrected for whole-brain analysis (FWE corrected, $<0.05$ ) in the dACC, bilateral aIns, bilateral PPC, and left lateral PFC. Within the right lateral PFC, significant FWE-corrected activation was also observed within a constrained region-of-interest contralateral to the leftsided, whole-brain-corrected activation (Figure 2, Table 3). Additional task-related activations were also in evidence in the bilateral extrastriate cortex and cerebellum.

Group-by-condition interactions within task-relevant cortical regions did not distinguish significant group differences in the magnitude of functional activations in any region, with or without controlling for the influence of alcohol consumption, tobacco use, history of other illicit drug abuse, RT, or current age. Lifetime cannabis use and age of regular cannabis-use onset were also not associated with task-related activity in the brain. However, greater indices of recent cannabis use were predictive of increased
Table 2 Group Mean Reaction Time and Median Accuracy Measures

\begin{tabular}{llc}
\hline & Controls $(\boldsymbol{n}=\mathbf{2 1})$ & Cannabis $(\boldsymbol{n}=\mathbf{2 1})$ \\
\hline Congruent RT, ms (SD) & $650.5(121.5)$ & $690.2(193.1)$ \\
Incongruent RT, ms (SD) & $969.3(132.3)$ & $1063.0(194.2)$ \\
Congruent accuracy, \% (range) & $100.0(95.8-100)$ & $100.0(97.9-100)$ \\
Incongruent accuracy, \% (range) & $97.9(91.7-100)$ & $95.8(89.6-100)$ \\
\hline
\end{tabular}

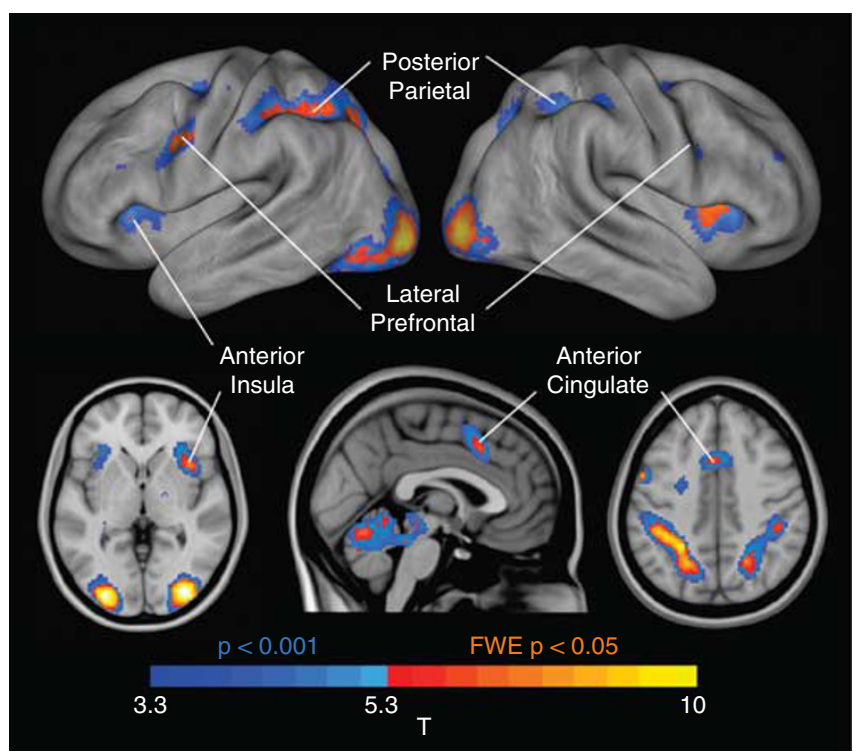

Figure 2 Main positive effect of condition. Warm colors indicate activations that meet the threshold for voxelwise familywise error (FWE) correction at a threshold of $p<0.05$, whereas cool colors represent additional activations at an uncorrected threshold of $p<0.001$. Labels highlight each of the seven regions-of-interest used in subsequent inference.

activations within small areas of the left lateral PFC (salivary delta-9-THC: $x=-56, y=0, z=32, T_{\max }=4.13, K_{\mathrm{E}}=31$; urinary carboxy-THC: $x=-58, y=18, z=22, T_{\max }=4.23$, $\left.K_{\mathrm{E}}=16\right)$.

\section{fMRI: Connectivity}

As presented in Figure 3 and Table 4, cognitive control demands were associated with stronger PPIs (ie, connectivity) in the cannabis group, as compared with controls, between each of the (i) dACC, (ii) left PFC, (iii) left aIns, and (iv) right aIns seed regions, and the left occipitoparietal cortex (OP) located at the junction of the intraparietal sulcus and dorsal extrastriate cortex (Figures $3 \mathrm{a}$ and $\mathrm{b}$ ). A between-group difference was also evident between the right lateral PFC seed region and the same area of the left OP at a slightly reduced statistical threshold $(p<0.002)$. Further, cannabis users also demonstrated relatively increased connectivity between the dACC seed region and the right dorsal extrastriate cortex. These group differences remained significant after accounting for alcohol and tobacco use, current age, behavioral performance, and history of 
Table 3 Positive Main Effect of Condition

\begin{tabular}{|c|c|c|c|c|c|c|}
\hline \multirow{2}{*}{ Region (BA) } & & \multirow{2}{*}{$K_{\mathrm{E}}$} & \multicolumn{3}{|c|}{ Coordinates } & \multirow{2}{*}{$T_{\max }$} \\
\hline & & & $x$ & $y$ & $\mathbf{z}$ & \\
\hline \multicolumn{7}{|c|}{ Voxel-wise FWE-corrected $p<0.05$ for whole-brain search space } \\
\hline Extrastriate cortex (I8/19) & $\mathrm{L}$ & 964 & -30 & -92 & -4 & 10.88 \\
\hline Extrastriate cortex (18/19) & $\mathrm{R}$ & 784 & 32 & -86 & -4 & 10.55 \\
\hline Superior parietal lobule (7) & L & 1284 & -26 & -48 & 42 & 8.55 \\
\hline Inferior parietal lobule (40) & & & -34 & -42 & 40 & 8.41 \\
\hline Inferior frontal junction (6/9) & L & 200 & -56 & 6 & 38 & 8.19 \\
\hline Anterior insula (13) & $\mathrm{R}$ & 212 & 34 & 20 & 4 & 7.57 \\
\hline Cerebellum, anterior lobe & $L$ & 239 & -28 & -54 & -26 & 7.49 \\
\hline Cerebellum, anterior lobe & $\mathrm{R}$ & 736 & 32 & -56 & -28 & 7.18 \\
\hline Inferior parietal lobule (40) & $\mathrm{R}$ & 78 & 44 & -36 & 44 & 6.67 \\
\hline Superior parietal lobule (7) & $\mathrm{R}$ & 170 & 22 & -60 & 42 & 6.5 \\
\hline Anterior cingulate cortex (32) & - & 104 & -2 & 14 & 42 & 6.49 \\
\hline Caudate tail & $\mathrm{L}$ & 3 & -16 & -20 & 20 & 5.93 \\
\hline Insula (13) & L & 19 & -32 & 24 & 0 & 5.9 \\
\hline Dorsal premotor cortex (6) & L & 12 & -24 & -4 & 54 & 5.62 \\
\hline Caudate tail & $\mathrm{R}$ & 4 & 24 & -36 & 14 & 5.51 \\
\hline
\end{tabular}

Small volume correction in right lateral prefrontal cortex ${ }^{\mathrm{a}}$

$\begin{array}{lllllll}\text { Inferior frontal junction (6/9) } & R & 147 & 42 & 6 & 26 & 5.06^{\mathrm{b}}\end{array}$

aSphere of $16-\mathrm{mm}$ radius centered at $56,6,38$, corresponding to contralateral activity.

${ }^{b}$ Significant at FWE-corrected $p<0.05$ within the search space, and $p=0.10$ for whole-brain.

Abbreviations: BA, brodmann area; $\mathrm{Ke}$, cluster size; $T_{\max }$, t-statistic

polydrug use. As depicted in Figure 3c, post-hoc exploration indicated significant positive condition effects (ie, increased connectivity alongside increased cognitive control demand) in the cannabis group at all connections, whereas in the control group, connectivity was either unmodulated (dACC-left OP, left PFC-left OP, left aIns-left OP, and right aIns-left OP) or significantly decreased (dACC-right $\mathrm{OP}$ and right PFC-left OP). Furthermore, connectivity was comparable between groups during congruent trials, but diverged during incongruent performance, as illustrated in Figures 3d and 4, suggesting that connectivity differences were principally driven by differing responses to changing cognitive control demands. Figure 4, in particular, depicts the change in connectivity (ie, regression slope) in the left PFC-left OP connection in two individuals, one from each cohort, whose results most closely replicate grouplevel findings.

A post-hoc region-of-interest analysis was also undertaken at the left OP to determine if significant betweengroup local activation differences would be apparent. The average contrast estimates $\left(\beta_{\text {incongruent }}-\beta_{\text {congruent }}\right)$ from this region across individuals were entered into a two-sample $t$-test; a trend towards a larger activation in the cannabis group was evident $\left(t_{40}=1.86, p=0.07\right)$. As such, all five frontal seed regions showed a cannabis-related hyperconnectivity with the left $\mathrm{OP}$, alongside a trend towards greater activation during cognitive control in the same region.
Additionally, with respect to connections exhibiting group differences in magnitude, positive correlations in the cannabis-using group were identified between the magnitude of right PFC-left OP connectivity and both age of onset and lifetime cannabis exposure, and between the magnitude of left PFC-left OP connectivity and age of onset. Biomarkers of recent cannabis use and severity of withdrawal symptoms were not predictive of connectivity strength (Table 4).

\section{DISCUSSION}

This study demonstrated that long-term, heavy cannabis use is associated with increased functional connectivity between multiple cognitive control regions of the frontal cortex, including the dorsal anterior cingulate, lateral prefrontal and anterior insular cortices, and the occipitoparietal cortex. Conversely, there were no significant group differences in the magnitude of brain activations during cognitive control in these same core regions of interest, or in task-related performance measures.

Observed increases in the strength of coupling occurred consistently in cannabis users between the frontal cognitive control regions and the dorsal extrastriate cortex proximal to the ventral intraparietal sulcus and the lateral parietooccipital sulcus. This region has been variously referred to as the OP (or parieto-occipital), visual area 6, or the dorsomedial visual area (Fattori et al, 2009; Galletti et al, 2005; Luppino et al, 2005). The OP is considered to have a pivotal role in the direction and switching of attention to spatial targets and target features, in guiding stimulus selection, and in tracking self-referential motion, with particular regard to the control of reaching and grasping (Desimone and Duncan, 1995; Galletti et al, 1999; Galletti et al, 2003; Yantis, 2008). Cognitive control theories postulate that when the current context presents a requirement for active control processes, such as in novel, challenging, or goal-directed situations, frontal 'executive' regions, including the anterior cingulate and dorsolateral prefrontal cortices, work in concert to modulate activity within relevant perceptual or motor pathways to bias information processing and response outcomes in favor of current goals (Botvinick et al, 2001; Desimone and Duncan, 1995; Egner and Hirsch, 2005a). 'Top-down' modulation therefore serves to optimize behavioral performance and utilization of cognitive resources. The observed task-induced hyperconnectivity may therefore represent increased top-down control influences on selective attention and visual-tracking mechanisms in cannabis users.

As PPIs do not predict directionality of the influence between interacting brain regions, the possibility that increased connectivity may alternatively represent greater bottom-up information flow from visual to frontal regions must also be considered. However, this interpretation is not well supported. As illustrated in Figure 4, increased connectivity in the cannabis group was indexed by an increase in the strength of the proportional relationship characterizing activity in the OP and frontal cortex. This connectivity change came alongside comparable functional activations between the groups in frontal regions and a trend towards greater activation in the cannabis group in the OP. This result would be predicted by a top-down perspective, in which 


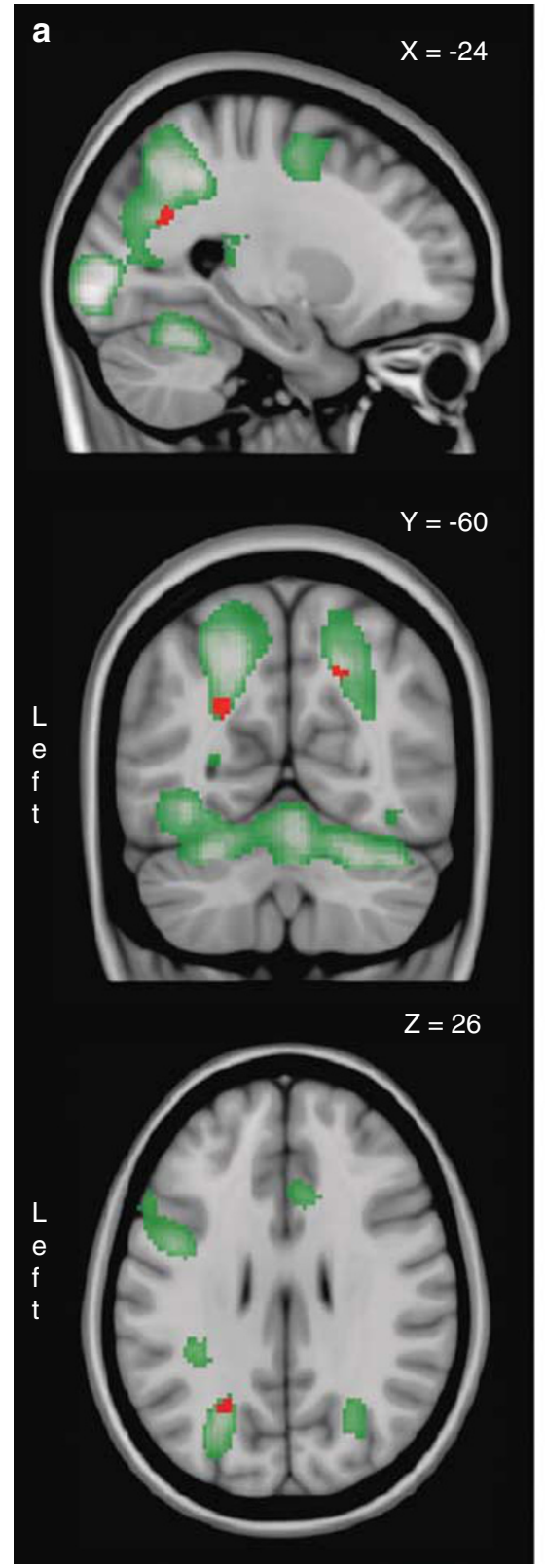

b

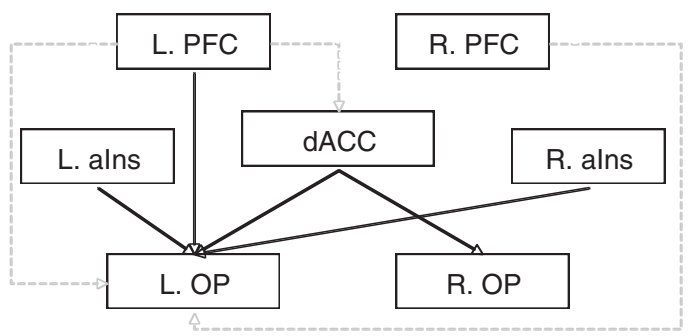

$\longrightarrow$ Hyperconnectivity $\quad \longrightarrow$ Cannabis Correlation
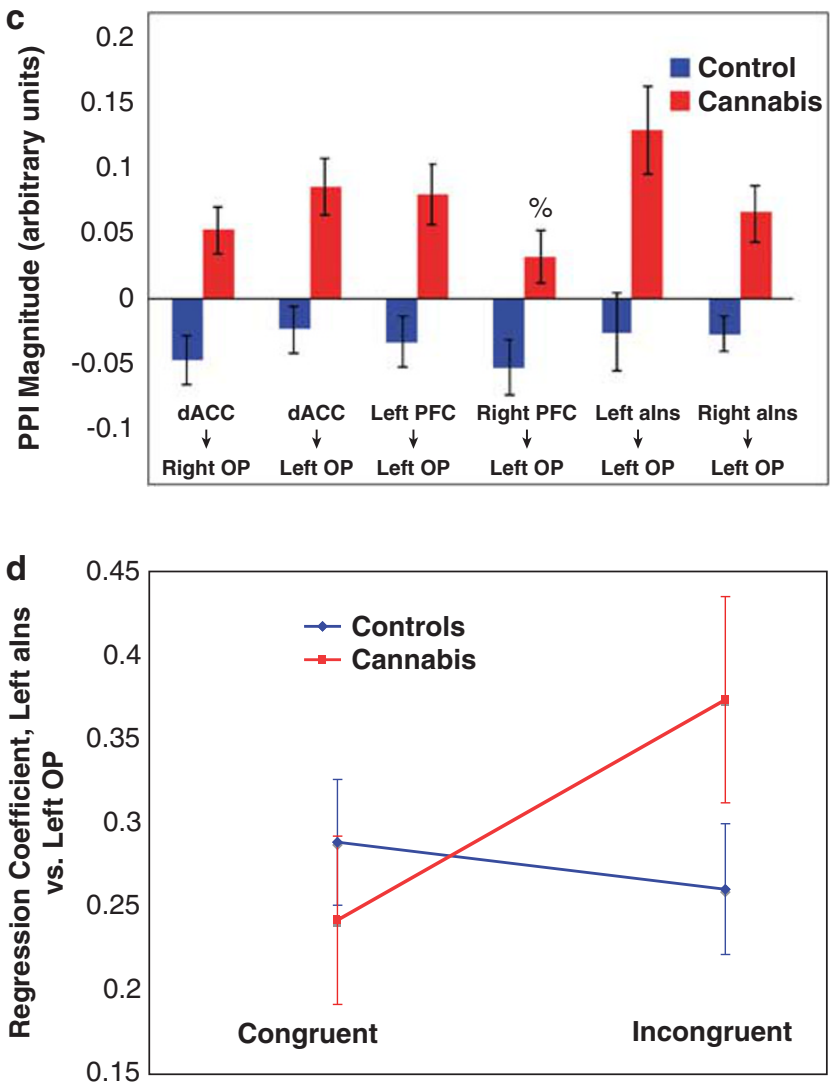

Figure 3 Increased functional connectivity in cannabis users from frontal lobe regions to the occipitoparietal (OP) cortex. The relevant region of the OP (red) and the main positive effect of condition (green) are overlayed onto sagittal, coronal, and axial brain slices (a; top to bottom). The corresponding circuit diagram depicts the connections in which a between-group difference is apparent, as well as those exhibiting a correlation with cannabis-use measures (b; $\mathrm{PFC}=$ prefrontal cortex; alns = anterior insula; $\mathrm{dACC}=$ dorsal anterior cingulate cortex). The magnitude of the group-averaged connection strengths is also depicted (c; error bars =SEM). The right PFC to left OP interaction has been included (\%) for completeness, even though this effect does not reach established significance thresholds. An interaction plot for the Left alns to Left OP connection is additionally included to highlight that group differences are driven by connectivity differences during incongruent processing (d; error bars $=$ SEM).

a comparable unit change in activity between the groups in the seed (ie, frontal) region, alongside a cannabis-specific increase in connectivity, would result in greater activation in the cannabis group in the target (ie, OP) region (Figure 4). From a bottom-up perspective, the slightly greater activation in the seed (ie, OP) region in cannabis users would be magnified by the increase in connectivity, resulting in a group activation difference in target (ie, frontal) regions as well. However, the latter eventuality was not observed. Furthermore, results were restricted to task-relevant brain regions; that is to say, regions that were activated by increases in cognitive control demands. As such, this region of the OP was likely engaged by mechanisms mediating cognitive control, specifically, the anterior cingulate and lateral prefrontal cortices (MacDonald et al, 2000; Ridderinkhof et al, 2004), and as such is not likely to be their precursor.

Accepting that fronto-occipitoparietal connections represent top-down control influences, the observed increases in connectivity may conceivably suggest that cannabis users require greater control over attentional and perceptual 


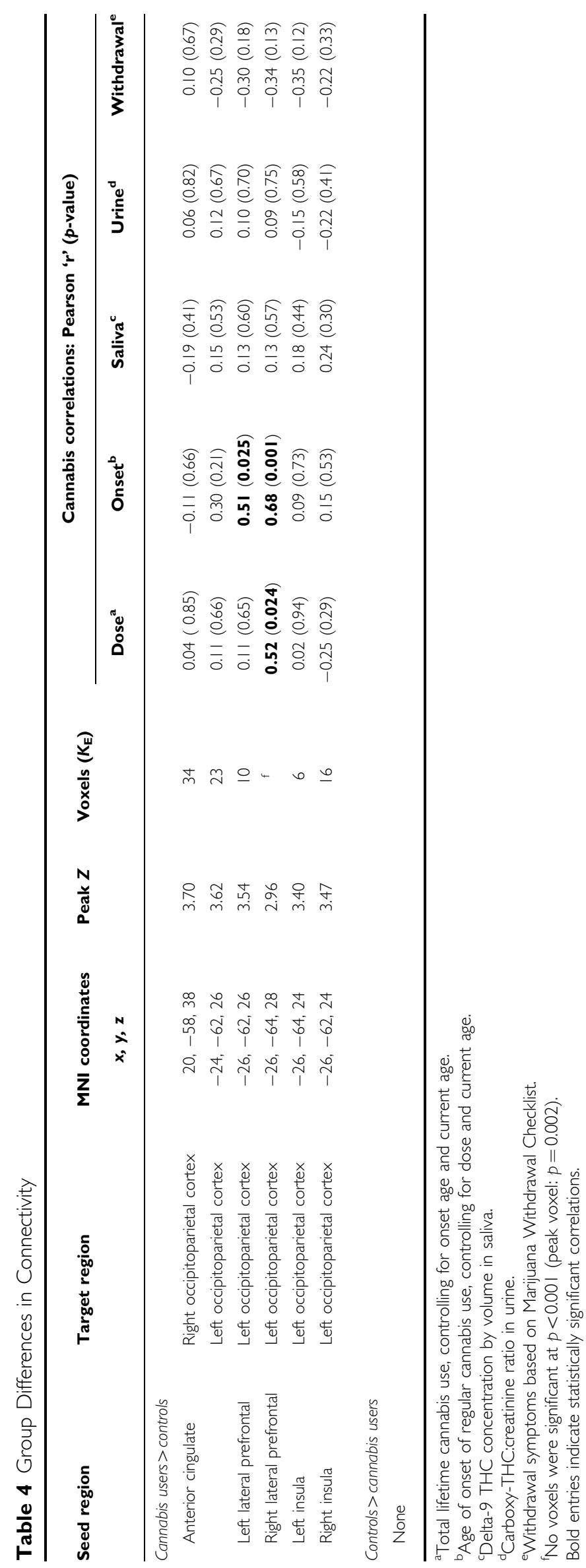

resources in cognitively demanding contexts. Acute cannabis intoxication has been linked to decreased occipital activity and disrupted perceptual processing during visual attention (Bocker et al, 2010; Weinstein et al, 2008), suggesting that cannabis may impair effective filtering and processing of sensory information. In addition, acute intoxication has been linked to hyperactivity in anterior cingulate, lateral prefrontal, and insular cortices during tasks of attention (O'Leary et al, 2007; Weinstein et al, 2008), suggesting either acute disruption of frontal attention and executive systems and/or the need for greater effortful activation of executive mechanisms to exact adaptive control over behavior. In response to chronic cannabis exposure, increased synaptic efficacy defining the interactions between prefrontal and occipital cortices may therefore compensate, at least partially, for deficits at either level (frontal executive or occipital perceptual) by magnifying the influence of control signals on perceptual processes. The presence of such an adaptive mechanism is consistent with the growing line of evidence indicating that heavier or longer-term users may become increasingly tolerant to the detrimental cognitive effects of intoxication, particularly within the domains of behavioral control and attention (Hart et al, 2001; Nordstrom and Hart, 2006; Ramaekers et al, 2009). In addition, as a result of varying degrees of progressive compensatory synaptic alteration within targeted networks, the findings of individual investigations exploring the impact of cannabis on cognitive control (and cognition in general) may be dependent on both the cohort (ie, long-term $v s$ short-term users) and/or task (ie, selective attention $v s$ response inhibition) under investigation. Such variance may help explain the current mixture of evidence for chronic cognitive impairments following extended cannabis use, with respect to both biological and behavioral indices of (dys)function (Crean et al, 2011; Martin-Santos et al, 2010).

Crucially, the magnitudes of task-induced connectivity change between several sets of task-relevant brain regions were also correlated with cannabis-use measures. The right prefrontal-to-left occipitoparietal connection strength was positively correlated with lifetime cannabis use. This result supports assertions argued above that increased cannabis use may be associated with greater compensatory changes in connectivity. Additionally, the magnitude of connectivity between the bilateral PFC and the left OP were positively associated with the age of onset of regular cannabis use. This finding indicates that a later age of onset was associated with greater differences in cortical connectivity within control networks. The age of onset in our sample ranged from 12 to 19 years of age (plus one outlier at 25 years), representing a cross-section of substantial maturation within the frontal and parietal association cortices of the brain (Gogtay et al, 2004), and continued development of top-down control mechanisms (Adleman et al, 2002; Luna et al, 2001; Rubia et al, 2000). It is therefore possible that neurodevelopmental trajectories are altered in younger starters in response to chronic cannabis exposure within the plastic brain, bearing in mind that this cohort maintained a level of near daily cannabis consumption from their onset through to the present. Conversely, the more mature brains of older starters may rely on alterations in synaptic efficacy once neural pathways are more 


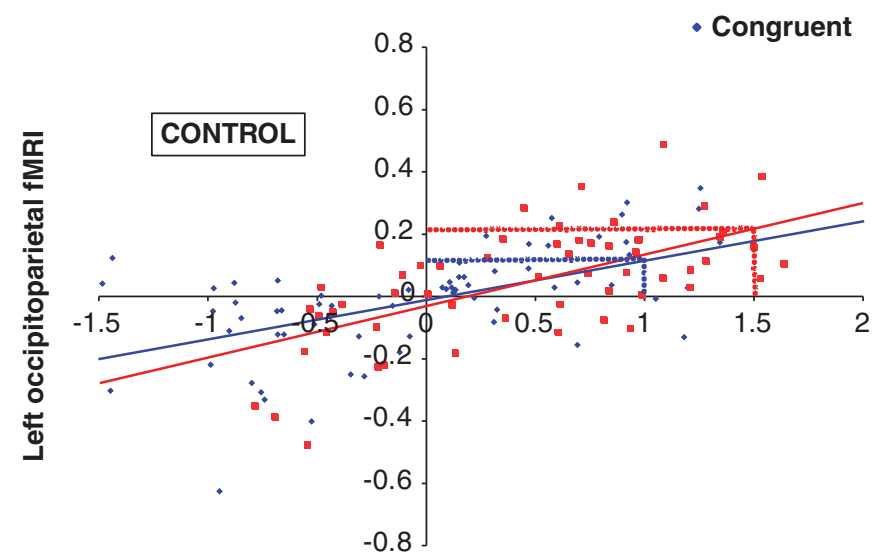

Left Prefrontal fMRI

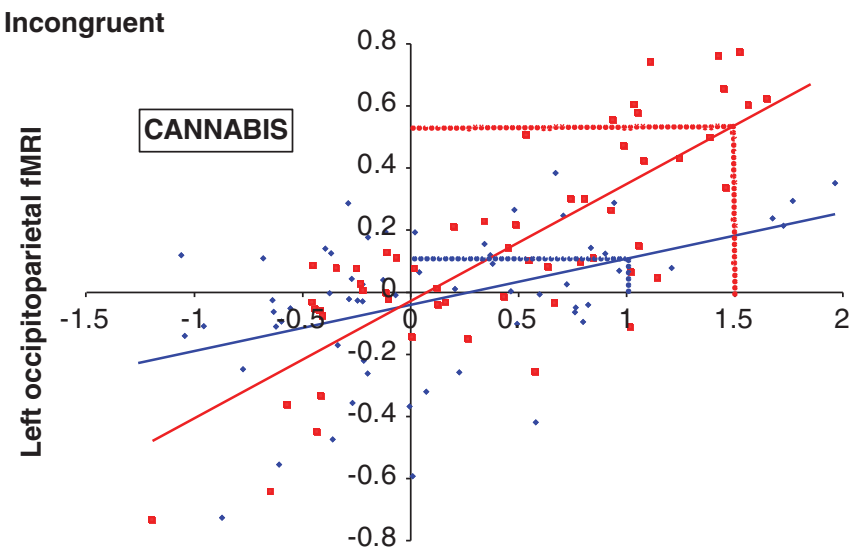

Left Prefrontal fMRI

Figure 4 Psychophysiological interactions between the left prefrontal and left occipitoparietal cortices in representative individuals from the 'CONTROL' (left) and 'CANNABIS' (right) groups. Each data point represents a functional magnetic resonance imaging (fMRI) signal measurement during congruent (blue) and incongruent (red) task performance, fitted with linear trend lines. Extrapolating to group-level results, the dotted lines illustrate how a comparable average unit change in activation between conditions in the seed region ( $x$ axis) can drive a between-group activation difference in the target region ( $y$ axis) due to group differences in connectivity change.

established. Put more simply, changes may be hard-wired in younger starters, whereas older starters invoke more dynamic compensatory adjustments in synaptic functioning. These findings are consistent with observations that cognitive deficits may be more persistent in younger starters following abstinence in later adulthood (Pope et al, 2003), and parallel reports that younger onset is associated with more detrimental cognitive outcomes (Jager and Ramsey, 2008; Solowij et al, 2011; Solowij et al, 2012).

\section{CONCLUSIONS}

In summary, we report increased functional connectivity between executive regions of the PFC and areas of the OP mediating the direction of attention in a cohort of current adult cannabis users during a task of cognitive control. We suggest that these findings may reflect a mechanism of compensatory top-down control mitigating abnormal attention and visual processing following chronic cannabis exposure. This is the first study to report connectivity disturbances underlying cognitive control in cannabis use, and among the first employing analysis of task-related functional connectivity to the study of illicit substance use.

Several limitations must be considered with respect to this study. The use of a retrospective approach limits the ability to make definitive causal inferences with respect to observed differences; future longitudinal studies will be necessary to disentangle the time course of connectivity changes with certainty. Additionally, in this study, we aimed to assess cannabis users in an unintoxicated state (greater than $12 \mathrm{~h}$ since last use), yet one in which withdrawal symptoms would not confound the results (within the first $24 \mathrm{~h}$ since last use; Budney et al, 2003). However, it is possible that observed discrepancies are the result of the residual effects of cannabis (see Crean et al, 2011 for review), rather than enduring dysfunction. Further research will be required to confirm the presence of similar effects after extended abstinence.

Future research extensions of this work include the application of functional connectivity analyses to additional cognitive domains and, due to the relevance of this work to understanding mechanisms of addiction and other harmful behaviors, to additional recreational substances. Such studies will help determine the relative specificity of findings and the longitudinal nature of effects (particularly with respect to long-term abstinence). Further experimentation exploring the role of top-down connectivity in addiction-related processes, such as selective attention biases, would also be valuable in understanding cognitive control deficits that may be specific to drug-relevant contexts.

\section{ACKNOWLEDGEMENTS}

This research was supported by funding from the National Health and Medical Research Council of Australia (NHMRC; Project Grant 459111). NS is supported by an Australian Research Council Future Fellowship (FT110100752). MY is supported by a NHMRC Fellowship Award (Grant 1001973). $\mathrm{BJH}$ is supported by an NHMRC Clinical Career Development Award (628509). CP is supported by an NHMRC Senior Principal Research Fellowship (Grant 628386) and NHMRC Program Grant (Grant 566529).

\section{DISCLOSURE}

DL has received a speaking honorarium from Astra Zeneca Pharmaceuticals, and $\mathrm{CP}$ has provided consultancy for Janssen-Cilag, Eli Lilly, Hospira (Mayne), and Astra Zeneca, Pfizer, Schering Plough, and Lundbeck, and has undertaken investigator-initiated studies supported by Eli Lilly, Hospira, Janssen Cilag, and Astra Zeneca. These activities are unrelated to the present work. Otherwise, except for income received from our primary employers and funding bodies listed in Acknowledgements, no financial support or compensation has been received from any individual or corporate entity over the past three years for research or professional service and there are no personal financial holdings that could be perceived as constituting a potential conflict of interest. 


\section{REFERENCES}

Abdullaev Y, Posner MI, Nunnally R, Dishion TJ (2010). Functional MRI evidence for inefficient attentional control in adolescent chronic cannabis abuse. Behav Brain Res 215: 45-57.

Adleman NE, Menon V, Blasey CM, White CD, Warsofsky IS, Glover GH et al (2002). A developmental fMRI study of the stroop color-word task. Neuroimage 16: 61-75.

Allen JP, Litten RZ, Fertig JB, Babor T (1997). A review of research on the alcohol use disorders identification test (AUDIT). Alcohol Clin Exp Res 21: 613-619.

Ashburner J (2007). A fast diffeomorphic image registration algorithm. Neuroimage 38: 95-113.

Battisti RA, Roodenrys S, Johnstone SJ, Pesa N, Hermens DF, Solowij N (2010). Chronic cannabis users show altered neurophysiological functioning on Stroop task conflict resolution. Psychopharmacology (Berl) 212: 613-624.

Bocker KB, Gerritsen J, Hunault CC, Kruidenier M, Mensinga TT, Kenemans JL (2010). Cannabis with high delta9-THC contents affects perception and visual selective attention acutely: an event-related potential study. Pharmacol Biochem Behav 96: 67-74.

Botvinick MM, Braver TS, Barch DM, Carter CS, Cohen JD (2001). Conflict monitoring and cognitive control. Psychol Rev 108: 624-652.

Budney AJ, Moore BA, Vandrey RG, Hughes JR (2003). The time course and significance of cannabis withdrawal. J Abnorm Psychol 112: 393-402.

Budney AJ, Novy PL, Hughes JR (1999). Marijuana withdrawal among adults seeking treatment for marijuana dependence. Addiction 94: 1311-1322.

Bush G, Shin LM (2006). The multi-source interference task: an fMRI task that reliably activates the cingulo-frontal-parietal cognitive/attention network. Nat Protoc 1: 308-313.

Cabeza R, Nyberg L (2000). Imaging cognition II: An empirical review of 275 PET and fMRI studies. J Cogn Neurosci 12: 1-47.

Cahn-Weiner DA, Farias ST, Julian L, Harvey DJ, Kramer JH, Reed $\mathrm{BR}$ et al (2007). Cognitive and neuroimaging predictors of instrumental activities of daily living. J Int Neuropsychol Soc 13: 747-757.

Chevaleyre V, Takahashi KA, Castillo PE (2006). Endocannabinoid-mediated synaptic plasticity in the CNS. Annu Rev Neurosci 29: 37-76.

Crean RD, Crane NA, Mason BJ (2011). An evidence based review of acute and long-term effects of cannabis use on executive cognitive functions. J Addict Med 5: 1-8.

Curran HV, Brignell C, Fletcher S, Middleton P, Henry J (2002). Cognitive and subjective dose-response effects of acute oral Delta 9-tetrahydrocannabinol (THC) in infrequent cannabis users. Psychopharmacology (Berl) 164: 61-70.

Desimone R, Duncan J (1995). Neural mechanisms of selective visual attention. Annu Rev Neurosci 18: 193-222.

Eggan SM, Lewis DA (2007). Immunocytochemical distribution of the cannabinoid CB1 receptor in the primate neocortex: a regional and laminar analysis. Cereb Cortex 17: 175-191.

Egner T, Hirsch J (2005a). Cognitive control mechanisms resolve conflict through cortical amplification of task-relevant information. Nat Neurosci 8: 1784-1790.

Egner T, Hirsch J (2005b). The neural correlates and functional integration of cognitive control in a Stroop task. Neuroimage 24: 539-547.

Eldreth DA, Matochik JA, Cadet JL, Bolla KI (2004). Abnormal brain activity in prefrontal brain regions in abstinent marijuana users. Neuroimage 23: 914-920.

Eriksen BA, Eriksen CW (1974). Effects of noise letters upon the identification of a target letter in a non-search task. Percept Psychophys 14: 155-160.

Fan J, Hof PR, Guise KG, Fossella JA, Posner MI (2008). The functional integration of the anterior cingulate cortex during conflict processing. Cereb Cortex 18: 796-805.
Fant RV, Heishman SJ, Bunker EB, Pickworth WB (1998). Acute and residual effects of marijuana in humans. Pharmacol Biochem Behav 60: 777-784.

Fattori P, Pitzalis S, Galletti C (2009). The cortical visual area V6 in macaque and human brains. J Physiol Paris 103: 88-97.

Fernandez-Serrano MJ, Perez-Garcia M, Schmidt Rio-Valle J, Verdejo-Garcia A (2010). Neuropsychological consequences of alcohol and drug abuse on different components of executive functions. J Psychopharmacol 24: 1317-1332.

Fillmore MT (2003). Drug abuse as a problem of impaired control: current approaches and findings. Behav Cogn Neurosci Rev 2: 179-197.

Friston KJ, Buechel C, Fink GR, Morris J, Rolls E, Dolan RJ (1997). Psychophysiological and modulatory interactions in neuroimaging. Neuroimage 6: 218-229.

Galletti C, Fattori P, Gamberini M, Kutz DF (1999). The cortical visual area V6: brain location and visual topography. Eur J Neurosci 11: 3922-3936.

Galletti C, Gamberini M, Kutz DF, Baldinotti I, Fattori P (2005). The relationship between $\mathrm{V} 6$ and $\mathrm{PO}$ in macaque extrastriate cortex. Eur J Neurosci 21: 959-970.

Galletti C, Kutz DF, Gamberini M, Breveglieri R, Fattori P (2003). Role of the medial parieto-occipital cortex in the control of reaching and grasping movements. Exp Brain Res 153: 158-170.

Gogtay N, Giedd JN, Lusk L, Hayashi KM, Greenstein D, Vaituzis AC et al (2004). Dynamic mapping of human cortical development during childhood through early adulthood. Proc Natl Acad Sci USA 101: 8174-8179.

Hart CL, van Gorp W, Haney M, Foltin RW, Fischman MW (2001). Effects of acute smoked marijuana on complex cognitive performance. Neuropsychopharmacology 25: 757-765.

Henry JD, von Hippel W, Baynes K (2009). Social inappropriateness, executive control, and aging. Psychol Aging 24: 239-244.

Hester R, Nestor L, Garavan H (2009). Impaired error awareness and anterior cingulate cortex hypoactivity in chronic cannabis users. Neuropsychopharmacology 34: 2450-2458.

Jacobsen LK, Pugh KR, Constable RT, Westerveld M, Mencl WE (2007). Functional correlates of verbal memory deficits emerging during nicotine withdrawal in abstinent adolescent cannabis users. Biol Psychiatry 61: 31-40.

Jager G, Ramsey NF (2008). Long-term consequences of adolescent cannabis exposure on the development of cognition, brain structure and function: an overview of animal and human research. Curr Drug Abuse Rev 1: 114-123.

Kano M, Ohno-Shosaku T, Hashimotodani Y, Uchigashima M, Watanabe M (2009). Endocannabinoid-mediated control of synaptic transmission. Physiol Rev 89: 309-380.

Konrad K, Eickhoff SB (2010). Is the ADHD brain wired differently? A review on structural and functional connectivity in attention deficit hyperactivity disorder. Hum Brain Mapp 31: 904-916.

Koob GF, Volkow ND (2010). Neurocircuitry of addiction. Neuropsychopharmacology 35: 217-238.

Lubman DI, Yucel M, Pantelis C (2004). Addiction, a condition of compulsive behaviour? Neuroimaging and neuropsychological evidence of inhibitory dysregulation. Addiction 99: 1491-1502.

Luna B, Thulborn KR, Munoz DP, Merriam EP, Garver KE, Minshew NJ et al (2001). Maturation of widely distributed brain function subserves cognitive development. Neuroimage 13: 786-793.

Luppino G, Hamed SB, Gamberini M, Matelli M, Galletti C (2005). Occipital (V6) and parietal (V6A) areas in the anterior wall of the parieto-occipital sulcus of the macaque: a cytoarchitectonic study. Eur J Neurosci 21: 3056-3076.

Lyons MJ, Bar JL, Panizzon MS, Toomey R, Eisen S, Xian H et al (2004). Neuropsychological consequences of regular marijuana use: a twin study. Psychol Med 34: 1239-1250. 
MacDonald 3rd AW, Cohen JD, Stenger VA, Carter CS (2000). Dissociating the role of the dorsolateral prefrontal and anterior cingulate cortex in cognitive control. Science 288: 1835-1838.

Maisto SA, Sobell LC, Cooper AM, Sobell MB (1982). Comparison of two techniques to obtain retrospective reports of drinking behavior from alcohol abusers. Addict Behav 7: 33-38.

Martin-Santos R, Fagundo AB, Crippa JA, Atakan Z, Bhattacharyya $S$, Allen $\mathrm{P}$ et al (2010). Neuroimaging in cannabis use: a systematic review of the literature. Psychol Med 40: 383-398.

Nee DE, Wager TD, Jonides J (2007). Interference resolution: insights from a meta-analysis of neuroimaging tasks. Cogn Affect Behav Neurosci 7: 1-17.

Nordstrom BR, Hart CL (2006). Assessing cognitive functioning in cannabis users: cannabis use history an important consideration. Neuropsychopharmacology 31: 2798-2799 author reply 2800-2791.

O'Leary DS, Block RI, Koeppel JA, Schultz SK, Magnotta VA, Ponto LB et al (2007). Effects of smoking marijuana on focal attention and brain blood flow. Hum Psychopharmacol 22: 135-148.

Pettersson-Yeo W, Allen P, Benetti S, McGuire P, Mechelli A (2011). Dysconnectivity in schizophrenia: where are we now? Neurosci Biobehav Rev 35: 1110-1124.

Pope Jr HG, Gruber AJ, Hudson JI, Cohane G, Huestis MA, Yurgelun-Todd D (2003). Early-onset cannabis use and cognitive deficits: what is the nature of the association? Drug Alcohol Depend 69: 303-310.

Pope Jr HG, Gruber AJ, Hudson JI, Huestis MA, Yurgelun-Todd D (2001). Neuropsychological performance in long-term cannabis users. Arch Gen Psychiatry 58: 909-915.

Ramaekers JG, Kauert G, Theunissen EL, Toennes SW, Moeller MR (2009). Neurocognitive performance during acute THC intoxication in heavy and occasional cannabis users. J Psychopharmacol 23: $266-277$.

Ridderinkhof KR, Ullsperger M, Crone EA, Nieuwenhuis S (2004). The role of the medial frontal cortex in cognitive control. Science 306: 443-447.

Rowe JB (2010). Connectivity analysis is essential to understand neurological disorders. Front Syst Neurosci 4: 144.

Rubia K, Overmeyer S, Taylor E, Brammer M, Williams SC, Simmons A et al (2000). Functional frontalisation with age: mapping neurodevelopmental trajectories with fMRI. Neurosci Biobehav Rev 24: 13-19.
Simon JR, Berbaum K (1990). Effect of conflicting cues on information processing: the 'Stroop effect' $v s$ the 'Simon effect'. Acta Psychol (Amst) 73: 159-170.

Solowij N, Jones KA, Rozman ME, Davis SM, Ciarrochi J, Heaven PC et al (2011). Verbal learning and memory in adolescent cannabis users, alcohol users and non-users. Psychopharmacology (Berl) 216: 131-144.

Solowij N, Jones KA, Rozman ME, Davis SM, Ciarrochi J, Heaven PC et al (2012). Reflection impulsivity in adolescent cannabis users: a comparison with alcohol-using and non-substanceusing adolescents. Psychopharmacology (Berl) 219: 575-586.

Solowij N, Stephens RS, Roffman RA, Babor T, Kadden R, Miller M et al (2002). Cognitive functioning of long-term heavy cannabis users seeking treatment. JAMA 287: 1123-1131.

Suchy Y (2009). Executive functioning: overview, assessment, and research issues for non-neuropsychologists. Ann Behav Med 37: 106-116.

Takagi M, Lubman DI, Cotton S, Fornito A, Baliz Y, Tucker A et al (2010). Executive control among adolescent inhalant and cannabis users. Drug Alcohol Rev 30: 629-637.

Tapert SF, Schweinsburg AD, Drummond SP, Paulus MP, Brown SA, Yang TT et al (2007). Functional MRI of inhibitory processing in abstinent adolescent marijuana users. Psychopharmacology (Berl) 194: 173-183.

Verdejo-Garcia AJ, Lopez-Torrecillas F, Aguilar de Arcos F, PerezGarcia M (2005). Differential effects of MDMA, cocaine, and cannabis use severity on distinctive components of the executive functions in polysubstance users: a multiple regression analysis. Addict Behav 30: 89-101.

Wang L, Liu X, Guise KG, Knight RT, Ghajar J, Fan J (2010). Effective connectivity of the fronto-parietal network during attentional control. J Cogn Neurosci 22: 543-553.

Weinstein A, Brickner O, Lerman H, Greemland M, Bloch M, Lester H et al (2008). Brain imaging study of the acute effects of Delta9tetrahydrocannabinol (THC) on attention and motor coordination in regular users of marijuana. Psychopharmacology (Berl) 196: 119-131.

Williams PG, Suchy Y, Rau HK (2009). Individual differences in executive functioning: implications for stress regulation. Ann Behav Med 37: 126-140.

Yantis S (2008). The neural basis of selective attention: cortical sources and targets of attentional modulation. Curr Dir Psychol Sci 17: 86-90. 